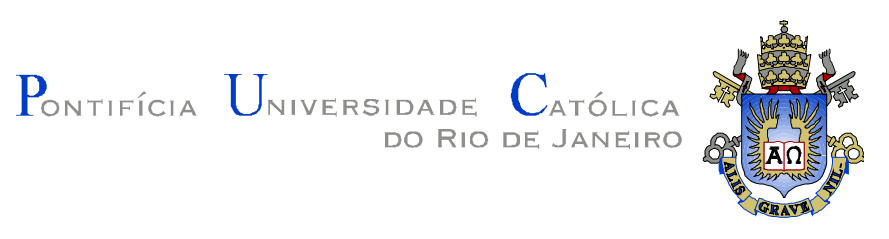

Mauricio Kreczmarsky Guimarães Meinicke

Compressão de dados sísmicos volumétricos utilizando a transformada wavelet 3D

Tese de Doutorado

Tese apresentada como requisito parcial para obtenção do título de Doutor pelo Programa de PósGraduação em Engenharia Mecânica da PUC-Rio.

Orientadores: Marcelo de Andrade Dreux

Pedro Mário Cruz e Silva

Rio de Janeiro

setembro de 2011 


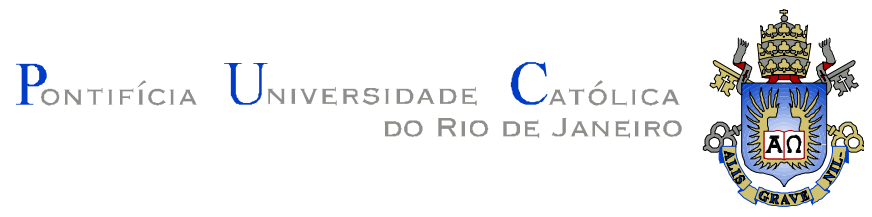

Mauricio Kreczmarsky Guimarães Meinicke

\title{
Compressão de dados sísmicos volumétricos utilizando a transformada wavelet 3D
}

\begin{abstract}
Tese apresentada como requisito parcial para obtenção do grau de Doutor pelo Programa de Pós-Graduação em Engenharia Mecânica do Centro Técnico Científico da PUC-Rio. Aprovada pela Comissão Examinadora abaixo assinada.
\end{abstract}

Prof. Marcelo de Andrade Dreux Orientador

Departamento de Engenharia Mecânica - PUC-Rio

Dr. Pedro Mário Cruz e Silva Orientador Tecgraf - PUC-Rio

Prof. Anselmo Cardoso Paiva Universidade Federal do Maranhão - UFMA

Prof. Bruno Feijó Departamento de Informática - PUC-Rio

Prof. David Sotelo Pinheiro da Silva Departamento de Informática - PUC-Rio

Prof. Luiz Eduardo Azambuja Sauerbronn Universidade Federal do Rio de Janeiro - UFRJ

Prof. José Eugenio Leal Coordenador(a) Setorial do Centro Técnico Científico - PUC-Rio 
Todos os direitos reservados. É proibida a reprodução total ou parcial do trabalho sem autorização da universidade, do autor e do orientador.

\section{Mauricio Kreczmarsky Guimarães Meinicke}

Graduou-se bacharel em Matemática pela Universidade do Estado do Rio de Janeiro (UERJ) em 2005. Mestre engenheiro mecânico pela Pontifícia Universidade Católica do Rio de Janeiro (PUC-RIO) na área de Mecânica Aplicada, em 2007.

Ficha Catalográfica

Meinicke, Mauricio Kreczmarsky Guimarães

Compressão de dados sísmicos volumétricos utilizando a transformada wavelet 3D / Mauricio Kreczmarsky Guimarães Meinicke; orientadores: Marcelo de Andradre Dreux e Pedro Mário Cruz e Silva - 2011.

94 fl. :il (color) ; $30 \mathrm{~cm}$

Tese (Doutorado em Engenharia Mecânica) Pontifícia Universidade Católica do Rio de Janeiro, Rio de Janeiro, 2011.

Incluí referências bibliográficas.

1. Engenharia Mecânica - Tese. 2. Compressão, 3. Dado Sísmico, 4. Wavelet 3D, 5. Transformada de Haar, 6. Atributo sísmico.

CDD: 621 


\section{Agradecimentos}

Aos meus pais pela paciência ao longo destes quatro anos. A todos os meus familiares pelo apoio neste projeto de vida. A minha namorada Regina.

Aos meus orientadores Marcelo Dreux e Pedro Mário, pela confiança e dedicação a este trabalho.

Aos professores do departamento de engenharia mecânica da PUC-Rio pelo apoio acadêmico necessário. Aos amigos de Pós-graduação.

A todos os amigos do Tecgraf/PUC-Rio que estiveram comigo nestes quatro anos. Um agradecimento especial aos envolvidos no projeto v3o2 pelo apoio e incentivo.

Ao $\mathrm{CNPq}$ e a PUC-Rio pelo auxilio financeiro. A Petrobras por permitir o desenvolvimento deste trabalho utilizando o software v3o2 de sua propriedade. 


\section{Resumo}

Meinicke, Mauricio Kreczmarsky Guimarães; Dreux, Marcelo; Silva, Pedro Mário Cruz; Compressão de dados sísmicos volumétricos utilizando a transformada wavelet 3D; Rio de Janeiro, 2011. 94p. Tese de Doutorado Departamento de Engenharia Mecânica, Pontifícia Universidade Católica do Rio de Janeiro.

A melhoria nas técnicas de obtenção de dados sísmicos fez com que estes dados sejam da ordem de até terabytes, o que gera um grande problema no armazenamento e transmissão dos mesmos. No que se refere ao problema de armazenamento em disco, apesar da queda no preço do disco rígido, o avanço das técnicas de obtenção do dado sísmico e o barateamento destas técnicas de obtenção do dado fazem com que a demanda por espaço em disco cresça mais rápido do que a queda no preço do hardware. Outro fator importante é a limitação de espaço físico para armazenar todos os discos rígidos de forma a garantir a segurança da informação destes discos. Este trabalho tem como objetivo apresentar uma nova técnica de compactação para dados sísmicos utilizando a transformada wavelet 3D. É feito um comparativo com as técnicas de compressão atuais, que utilizam a transformada $2 \mathrm{D}$.

\section{Palavras-chave}

Compressão; dado sísmico; Wavelet 3D; transformada de Haar; Atributo sísmico. 


\section{Abstract}

Meinicke, Mauricio Kreczmarsky Guimarães; Dreux, Marcelo (Advisor); Silva, Pedro Mário Cruz (Advisor); Volumetric seismic data compression using 3D wavelet transform; Rio de Janeiro, 2011. 94p. Dsc. thesis Departamento de Engenharia Mecânica, Pontifícia Universidade Católica do Rio de Janeiro.

Improvements made in techniques for seismic data acquisition have led to data that are of the order of terabytes, which in turn creates the problem of data storage and transmission of them through the network. In regards to the problem of disk storage, despite the hard disk decreasing price, the improvement and reduction cost in seismic data acquisition techniques has pushed the demand for disk space to grow faster than the reduction of the hardware cost. Another important factor is the space necessary to store all hard drives, to provide security for information from these records. In order to tackle these problems this thesis presents a new technique for seismic data compression using 3D wavelet transform. It is also presented in comparison with current compression techniques which use the $2 \mathrm{D}$ transform.

\section{Key-words}

Compression data; seismic data; 3D Wavelet; Haar transform; seismic attribute. 


\section{Sumário}

1 Introdução 14

1.1. Estrutura da Tese 16

2 Conceitos Básicos 18

2.1. Sísmica 18

2.1.1. Atributos Sísmicos 20

2.2. Transformada Wavelet 25

2.3. Quantização 31

2.4. Algoritmos de Compressão 32

2.4.1. Algoritmo de Huffman 34

2.4.2. Run Length Encode (RLE) 37

3 Trabalhos Relacionados 38

4 Método Proposto 52

4.1. Visão geral do Método 52

4.1.1. Transformada Wavelet 53

4.1.2. Quantização 57

4.1.3. Compressão 58

4.1.4. Descompressão 59

4.2. Estudo sobre Compressão de Atributos Sísmicos 60

4.2.1. Decomposição em atributos sísmicos 60

4.2.2. Compressão dos Atributos Sísmicos 68

5 Experimentos e Resultados $\quad 74$

5.1. Ordem dos algoritmos de compressão 75

5.2. Comparativo: Wavelet 2D x Wavelet 3D 77

5.3. Separar em Atributos 83

6 Conclusões e Trabalhos futuros $\quad 88$ 
6.1. Trabalhos Futuros $\quad 89$

6.1.1. Wavelet 3D 89

6.1.2. Uso do traço complexo 90

7 Referências Bibliográficas 92 


\section{Lista de figuras}

Figura 1 Aquisição de um dado sísmico marítimo. Adaptado de [17]

Figura 2 Volume post-stack com fatias, horizonte e probe (subregião de interesse). 20

Figura 3 Amplitude sísmica de um dado. 23

Figura 4 Envelope calculado sobre a seção sísmica representada na figura .

Figura 5 Fase calculada sobre seção sísmica representada na figura .

Figura 6 Frequência calculada sobre a seção sísmica representada na Figura .

Figura 7 Decomposição de Haar padrão aplicada as linhas da imagem.

Figura 8 Decomposição de Haar padrão aplicada as colunas da imagem.

Figura 9 Decomposição de Haar não padrão.

Figura 10 Diferentes níveis de resolução da imagem. representa a imagem original com 512 × 512 pixel. representa um nível de decomposição, sendo a imagem de 256 x 256 pixels. (c) representa a imagem com $128 \times 128$ pixels. (d) representa a imagem com $64 \times 64$ pixels.

Figura 11 Quantização escalar de um sinal.

Figura 12 Quantização vetorial. Adaptado de [28].

Figura 13 Arvore binária gerada na codificação de Huffman

Figura 14 Resultado da compressão de dados utilizando o método descrito por Averbuch et al. Adaptado de [3].

Figura 15 Comparativo entre o método proposto por Wu et al (WPC) e o método baseado na transformada wavelet tradicional (EZW). Adaptado de [4].

Figura 16 Padrão de sub-bandas da symmetric wavelet 
transform do padrão WSQ. Adaptado de [32].

Figura 17 Imagem de Impressão digital decomposta de acordo com o padrão WSQ definido pelo FBI. Adaptado de [32].

Figura 18 (a) Imagem original, disponível em [38]. (b) Magnitude espectral de (a). (c) Fase de (a)

Figura 19 (a) imagem original, disponível em [38]. (b) Magnitude espectral de (a). (c) Fase de (a).

Figura 20 (a) Imagem reconstruída através da fase da figura 18 e da magnitude espectral da figura 19. (b) Imagem reconstruída através da fase da figura 19 e da magnitude espectral da figura 18.

Figura 21 Transformada Wavelet 3D utilizada por Wang e Huang. Adaptado de [40].

Figura 22 Comparativo entre a taxa de compressão e PSNR para a transformada wavelet $2 \mathrm{D}$ e $3 \mathrm{D}$. (a) Tomografia computadorizada com intervalo de 5 milímetros entre as seções, (b) ressonância magnética com intervalo de 3 milímetros entre as amostras. Adaptado de [40].

Figura 23 Método convencional de compressão de dados sísmicos.

Figura 24 Dados sísmico Volume1.

Figura 25 Transformada de Haar 3D (a) e a 2D (b).

Figura 26 Duas seções sísmicas do Volume1 sobre o atributo de envelope.

Figura 27 Duas seções sísmicas do Volume1 sobre o atributo de fase instantânea.

Figura 28 Duas seções sísmicas do Volume2 sobre o atributo de amplitude sísmica.

Figura 29 Duas seções sísmicas do Volume2 sobre o atributo de envelope.

Figura 30 Duas seções sísmicas do Volume2 sobre o atributo de fase instantânea.

Figura $31 \quad$ (a) Volume1 reconstruídos, (b) Volume2 
reconstruído.

Figura 32 Seção sísmica do atributo de amplitude. 66

Figura 33 Seção sísmica sobre o atributo de envelope. 66

Figura 34 Seção sísmica sobre o atributo de fase instantânea. $\quad 67$

Figura 35 Seção sísmica sobre o atributo de frequência instantânea.

Figura 36 Compressão de dados sísmicos utilizando o traço complexo.

Figura 37 Volume1 decomposto utilizando a transformada wavelet 2D nas seções de crossline. 


\section{Lista de tabelas}

Tabela 1 Codificação de gerada para a árvore representada na figura 13.

Tabela 2 Comparativo entre as equações de atributos de imagens e dados sísmicos.

Tabela 3 Comparação entre as entropias dos atributos sísmicos.

Tabela 4 Comparação entre a entropia do dado original, com o uso das transformadas wavelet 3D e wavelet 2D.

Tabela 5 Comparação entre a entropia do dado quantizado e o uso da transformada wavelet junto com a quantização. 


\section{Lista de gráficos}

Gráfico 1 Curva de variação da taxa de compressão de acordo com o valor de $\mathrm{Zk}$.

Gráfico 2 Comparação da curva de taxa de compressão x SNR de atributos sísmico. O eixo horizontal representa a taxa de compressão obtida e o eixo vertical o erro da reconstrução do dado.

Gráfico 3 Comparativo entre a ordem de execução dos algoritmos de Huffman e RLE no dado sísmico Volume1.

Gráfico 4 Comparativo entre a ordem de execução dos algoritmos de Huffman e RLE no dado sísmico Volume3.

Gráfico 5 Curvas de compressão para 0 dado sísmico Volume1.

Gráfico 6 Curvas de compressão para o dado sísmico Volume2.

Gráfico 7 Curvas de compressão para 0 dado sísmico Volume3.

Gráfico 8 Comparativo da transformada wavelet 3D e 2D para dados sísmicos cujas dimensões não são potência de dois.

Gráfico 9 Comparativo da transformada wavelet para dado sísmico não cubo e com fundo do Mar.

Gráfico 10 Curva de compressão entre os atributos sísmicos do dado Volume2.

Gráfico 11 Comparativo entre a quantização de 256 classes e de 2048 classes.

Gráfico 12 Comparativo entre a taxa de compressão da combinação de atributos sísmicos para o volume1.

Gráfico 13 Comparativo entre a taxa de compressão da combinação de atributos sísmicos para o volume2. 